\title{
Marginal Deep Architectures Based Massive MIMO Beam Forming and Predictions
}

\author{
${ }^{1}$ Mithra Venkatesan, ${ }^{2}$ Anju.V.Kulkarni, ${ }^{3}$ Radhika Menon \\ ${ }^{1,2}$ E\&TC Department, D.Y.Patil Institute of Technology, Pimpri, Pune-411018 \\ mithra.venkatesan@gmail.com,anju_k64@yahoo.co.in \\ ${ }^{3}$ Maths Department, D.Y.Patil Institute of Technology, Pimpri, Pune-411018 \\ radhika.tharoor@gmail.com
}

Received: 30th May 2020, Accepted: 19th June 2020, Published: 31st August 2020

\begin{abstract}
The increasing demands in 5G technology is fulfilled by MIMO systems which employ hundreds of antennas to serve the broadband terminals. Deep learning algorithms are powerful machine learning algorithms capable of advanced learning and predictions. Deep learning is largely employed in MIMO. This paper explores the usage of deep learning architectures in beam forming and predictions in MIMO. Specifically, an improvised version of deep learning is the marginal deep architecture. This paper also briefs about the usage of marginal deep architecture based methods for beam forming and predictions. Such proposed models will greatly improve the efficiency and capability of beam forming in MIMO resulting in improved 5G services.
\end{abstract}

Keywords

5G, MIMO, Deep Learning, Beam Forming

\section{Introduction to MIMO}

There is tremendous growth of mobile data applications along with increasing expectations from $5 \mathrm{G}$ technologies. This has in turn resulted in the great requirement for wireless networks to increase its capacity. There are predictions that three major technologies are going to play a major role in empowering $5 \mathrm{G}$ technology and help in realising the full potential of $5 \mathrm{G}$. One is the usage of carrier frequencies in the range 10 to $100 \mathrm{GHz}$ called the millimetre band. The second is the densification of the network by small sized cells or femto cells. The third technology is MIMO.Towards implementing 5G technology Multiple Input Multiple Output (MIMO) technique will play a major role. [1] describes the various aspects associated with MIMO including the antenna design, presence as array, its configuration and challenges.

MIMO systems make use of hundreds of antennas to improve performance along with ability to serve number of broadband terminals. Robust signal processing methods are made use of in Massive MIMO along with precise beam forming techniques. [2] presents complete review of homogeneous and heterogeneous MIMO looking into its key components, advantages and disadvantages.[3] focuses on MIMO technology which has been extended in usage where micro wave and mm-wave frequencies are used. The six basic differences where MIMO is used in both these frequencies are looked into. The difference in architecture, algorithms along with the possible performances that can be obtained in different frequencies is presented in this work.[4] discusses the position and power levels of base stations in MIMO system towards realising reduced power consumption, low downlink and uplink exposure to electromagnetic interferences and improved user coverage. The system is practically implemented in Belgium with larger number of base station antenna elements and lesser number of base stations required. The statistical results show improved performance. Multiple antenna application is an essential constituent of 5G technology and can achieve improved performance through different approaches. [5] presents a survey of three different methods through which this can be accomplished namely Cell free Massive MIMO, beam space Massive MIMO and intelligent reflecting surfaces. The motivation and salient features of these technologies are discussed in the paper.

There is limitation of number of antennas that can be equipped in base station acting as a limiting factor for the deployment of massive linear arrays. To overcome this challenge [6] discusses Full Dimension MIMO (FDMIMO) which utilises an Active Antenna System (AAS) which has the capacity to perform adaptive electronic beam forming. The results of the effectiveness of the method are demonstrated in 5G FD-MIMO systems. There are different standardisation methods which can be used in MIMO. [7] explores the different standardisation techniques along with different methods which can be used for channel modelling and measurements. Channel estimation methods leading to precoding and signal detection is summarised. The use of Massive antenna arrays in $5 \mathrm{G}$ is done through usage of various spatial signatures of users. This feature can also be utilised to find the location of the users. Hence [8] explores the various localisation methods and refined channel estimation techniques to do the task of massive MIMO localisation which is fast emerging domain. Clustering is effective in Massive MIMO to organise network topology towards enhancing network level and cluster level performance metrics. [9] presents a complete review of different methods of clustering along with its characteristics, metrics and challenges. 
Massive MIMO could be successful in achieving high spectral efficiency and low latency if there are good MIMO detectors present. There are number of MIMO detection algorithms available. [10] presents a complete survey of various MIMO detection algorithms for small and medium dimesion MIMO systems. From the review it is understood that there is no single ideal MIMO detector and every MIMO detection algorithms has its advantages and disadvantages.

\section{Usage of Deep Learning in MIMO}

Because of its computational efficiency and versatility Machine Learning has found major applications in 5G technology enabling the networks become robust. Deep Learning methods in Machine Learning has wide range of usage in 5G.[11] reviews the development of deep learning solutions for 5G communication. The paper also discusses effcient deep learning based schemes for MIMO and mmWave based systems. The novel communication framework called NOMA is explored in tha paper along with its superior performance being investigated.[12] investigates the usage of Jrip, J48 and Naïve bayes machine learning algorithms to predict transmission states in a channel in $5 \mathrm{G}$ networks. The database required for this is produced by recreations on the channel condition.[13] reviews different techniques involved in device to device communication in $5 \mathrm{G}$ networks and explores the usage of artifical intelligence based device to device communication. The AI based schemes have better interference mitigation and superior resource allocation compared to conventional methods.

Load balancing and control with interference mitigation in $5 \mathrm{G}$ networks is explored through AI based particle swarm optimisation in [14]. To reduce the interference problem, joint transmission coordinated multipoint is being used that are present in cell expansion area.It is being observed that this method brings improvement in throughput associated with the system. Interference mitigation can also be perfomed through learning in changing Internet of Things environment. This is exhibited in [15] where the proposed policy has the ability to adapt itelf quickly and efficeintly to the changes in the network and depends only on the locally available information. The obtained results indicate that the interference due to the connecting devices is greatly reduced in an adaptive, distributed fashion in this methodology.

\section{Beamforming in MIMO}

In Massive MIMO multiple antennas are being deployed. Beam forming is the technique used with multiple antennas to control the direction of a wavefront by approximately finding the magnitude and phase of the individual antenna signals in the array of multiple antennas. Massive MIMO along with beam forming antenna array technology play a significant role in 5G communication. [16] presents a review of various types of beam forming techniques and the techniques most suitable for usage in massive MIMO systems. A new technique of optimal beamforming is suggested in the paper capable of good performance in massive MIMO environment.

[17] discusses different salient implementation issues which arise towards deploying of MIMO processing in 5G systems. The different architectures for MIMO is being described where superior performance is obtained using hybrid approach. All the implementation is being carried out for multi user MIMO transmissions carried out in millimetre wave bands.[18] discusses the numerical evaluation of ZF and MMSE type beam forming and NOMA in indoor lab environment based practical massive MIMO systems. The base station has 4 antennas and 4 users. The performance is evaluated under different conditions. Hybrid beam forming for MIMO system is looked into under jamming environment [19]. The proposed method has ability to reduce the covariance matrix and hence better interference mitigation. Simulations have been performed to confirm the effectiveness of the proposed method. The key implementation issues in Massive MIMO beam forming in 5G systems are being discussed. [20] Both performance and implementation issues are being detailed with description of MIMO architecture for the same. Hybrid analog and digital beam forming designs based on single chain architecture for massive MIMO are elucidated in [21]. Three new beam former designs are being proposed which overcomes the shortcomings of the described method. The applications of the three methods proposed are also analysed. Simulations also support the superiority of the proposed methods.

\section{Methodology \\ Use of Deep Learning in Beamforming in MIMO}

There are many challenges in working MIMO in mmWave systems such as impact on coverage, reliability and need for frequent hand-off between base stations. The finding of optimal beam forming also becomes challenging under these situations. To overcome these difficulties [22] proposes an innovative method integrating machine learning techniques with beamforming. The proposed method makes use of deep learning models to predict beamforming vectors at the base stations. This yields a complete solution supporting mmWave applications with improved performances.

[23] explores a deep learning enabled mmWave massive MIMO framework for effective hybrid precoding. Deep neural network based approach has reduced bit error ratio and improved spectrum efficiency and has exhibited better performance and reduced complexity compared to conventional schemes. Due to great increase in data volume, 5G networks are becoming more complex in terms of deployment. Hence [24] proposes a novel algorithm involving combination of three neural network towards optimisation of performance of massive MIMO beamforming. The first neural network generates user mobility pattern. The second neural network is used to 
generate antenna diagrm, while the third set of neural network is used to find the efficiency of the generated antenna diagram. The major advantage of the proposed method is that it doesnot require large training sets.

The complexity of cellular network calls in for the usage of deep learning algorithms in $5 \mathrm{G}$ networks.However there is scarcity of large datasets which prevents the usage of deep learning techniques in 5G networks. [25] explores the usage of specific datasets towards finding beam selection technique or mmWaves. The usage of deep learning and reinforcement learning on the database helps realsie beam forming selection in massive MIMO $5 \mathrm{G}$ networks.Hybrid beamforming is a potential answer for massive MIMO systems. [26] proposes anolg beam selection method based on machine learning. The training dataset in this case is made of huge number of samples off the mmWave channel. Support Vector Machine algorithm has been used to maximise the sum rate.A novel method has also been proposed to find the optimal parameter.It has been established that the complexity has been reduced substantially using the proposed method.

[27] discusses the usage of Multilayer perceptron mechanism model in massive MIMO systems. There are two models used, one for beamforming and the other is used for channel estimation. The output from the beamforming MLP is given as input towards channel estimation.Mean square error and bit error rate are used as performance metrics and the system developed exhibits superior performance.The use of deep learning algorithms reduce the number of epochs and capable of predicting in real time with high efficiency. In MIMO systems, obtaining inormation on downlink channel state information is very difficult due to increased overheads in uplink feedback and downlink training. In order to solve the issue [28] proposes a sparse complex valued neural network(ScNet) to approximate the uplink to downlink mapping function. This ScNet used exhibits better performance accuracy along with improved robustness for complex wireless channels making it a potential solution for use in practical deployment.[29] describes the usage of deep learning in performing power alloctaion in downlink of Massive MIMo networks. The trained deep neural network is capable of learning the mapping between user equipments and power allocation policies. Subsequently, it is used to predict the power allocation polices. The usage of deep neural network makes the system perform better optimisation compared to conventional methods and the proposed method doesnot require any statistical averages.

.Multi user detection algorithms are essential and play a major role in Massive MIMO OFDM systems.[30] describes a combination of channel estimation and multi user detection for removing interferences and reducing Bit Error dats. Such a model is proposed by the usage of sparse based k-neighbour classifier.Activity detection and pilot placement are inherent part of the work which paves way to reduced mean square error, symbol error rate and bit error rate.[31] discusses an unspervised deep learning based physical layer scheme for single user MIMO system. This method makes use of an autoencoder.Rayleigh fading channel model is introduced into the autoencoder to learn a system which optimises it. The proposed system can easily adapt itself for both open loop and closed loop operations in multiplexing modes and spatial diversity. The method's performance is superior compared to conventional method.

In $5 \mathrm{G}$ non-orthogonal multiple access is quite popular in the field of wireless communication. Successive interference cancellation is the main NOMA detection method used. [32] explores a deep learning method that automatically analyses the channel state information and finds the original transmit sequence. The deep learning method combines this channel estimation along with recovery of desired signal affected by channel distortion and signal superposition. In contrast to SIC method, deep learning methods addresses channel impairment problems and achives good performance.It is found that deep learning is an effcient tool for signal detection.[33] proposes a deep neural network based hybrid beamforming for multiuser massive MIMO systems. The system is being trained using self-supervised algorithms. Through simulations it is established that the proposed method outperforms in terms of bit error rate compared to the existing traditional methods.[34] details beam allocation problem for massive MIMO sysem using deep neural networks.Beam selection and switching is performed using machine learning algorithms. Beams are produced by Butler metod to achieve large gain. The base stations are being interpreted by multiclass classification. Simulation results exhibit that the proposed scheme is able to forecast and allocate beams with good accuracy providing superior communication performance to the receivers. [35] details a deep learning based framework for performing two functions namely hybrid beamforming as well as joint antenna selection. Two convolutional neural networks are being used, which takes in input as channel matrix and give antenna subarray as the output. A part of the channel matrix is given to second CNN and its output is given to both analog and digital beamformers. Simulations performed indicate that CNN framework exhibits better performance compared to basic techniques.

In order to handle the deviations in the channel characteristics and provide robust perfromance, deep learning approach Convolution Neural Network with quantised weights is proposed[36]. The purpose of these quantised weights is to run these algorithms on mobile devices also with less available memory. The proposed methos saves a minimum of 6 bits and displays good performance in contrast to conventional MIMO hybrid beamforming techniques.Hot spot predictions based virtual small cell improves te operating effciency in 5G systems. This hot spot prediction is being performed through deep learning and this in turn aids in adaptive beamforming as exhibited in [37].Feature extraction capability of deep learning and long short term memory of neural networks is being utilised to implement the required functionality.Large scale antenna array with hybrid beamforming is 
adaptively adjusted to cover high transmission across hotspot of the virtual small cells. The deployment of the method greatly improves energy efficiency and reduces latency.Antenna selection and hybrid beamforming design is solved as a classification and prediction problem using Convolution neural networks as in[38].The training of the convolutional neural networks are being carried out under noisy channel matrices and different channel statistics to achieve improved performances. The usage of CNN results in improved spectral effciency and qauntised weights usage results in saving of bits making the system suitable for mobile devices.

There are different variants of deep models which are being used for varied applications such as image classification, speech recognition etc. [39] proposes to stack feature learning modules towards the design of the deep architectures. Marginal Fisher analysis is being stacked in this proposed architecture and hence called Marginal deep Architecture. The method combines different deep learning techniques in order ro fine tune the model. Extensive experimentations for different applications leads to conclusions that MDA is better than not only shallow feature learning models but also certain deep learning models.[40] discusses stacked feature learning model involving layer by layer initialisation of the deep architecture called Marginal Deep Architectures. The effectiveness of the proposed model is displayed for small and middle scale real world applications as the model doesnot require large training data [41] explores object detection and segmentation in context of image parsing using Marginal Space deep learning. The run time performance of the system is greatly improved along wit computational efficiency. The potential of Marginal Spce Deep Learning is fully exploited towards solving 3D data with parametrised representations.

Based on the literature survey done, it is understood that beamforming plays an important role in MIMO systems. There are various algorithms and methods to accomplish beamforming. It is also observed that Deep Learning based hybrid beamforming is more effective because of reduction in computational complexity and improved efficiency. Marginal Deep Architectures combine different deep learning methods to fine tune the model. Hence the proposed work in this paper is towards developing a Marginal Deep Architecture based model for Massive MIMO beeamforming and predictions.

\section{Result and Discussion}

\section{Proposed Marginal Deep Architectures for Massive MIMO Beam Predictions}

In order to implement the proposed marginal deep architecture for massive MIMO beam predictions, there is required of standard dataset.In fact, different machine learning algorithms are increasingly finding usage in Massive MIMO and mmWave applications. However the absence of a common dataset for all the algorithms is a major drawback in validating different developed algorithms. Towards the same [42] explores the developed generic database which has been developed for different massive MIMO and mmWave applications. The paper describes the methodology used in generating the database through outdoor ray tracing scenario with 18 base stations and more than 1 million users. The application of this generated database in MIMO applications is also briefed.The paper also describes a novel integrated machine learning and coordinated beamforming solution where a number of distributed coordinating base stations simultaneously serve a mobile user. This model has been developed for highly mobile mm-Wave application.

\section{Conclusions}

This paper gives a review on MIMO systems, application of deep learning in MIMO systems, beam forming in MIMO and beam forming in MIMO using deep learning architectures. The paper also gives insights on Marginal deep architectures and how marginal deep architectures can be potentially used in beamforming in MIMO towards improving computational complexity and efficiency. The details of the standard database is to be used for the same is also discussed.

\section{References}

1. H. M. El Misilmani and A. M. El-Hajj, "Massive MIMO Design for 5G Networks: An Overview on Alternative Antenna Configurations and Channel Model Challenges," 2017 International Conference on High Performance Computing \& Simulation (HPCS), Genoa, 2017, pp. 288-294, doi: 10.1109/HPCS.2017.52.

2. Noha Hassan and Xavier Fernando, Massive MIMO Wireless Networks: An Overview, Electronics,2017,Vol.6, Issue 63.

3. Stefano Buzzi, Carmen D'Andrea, Massive MIMO 5G Cellular Networks: mm-wave vs. $\mu$-wave Frequencies, arXiv preprint arXiv: $1702.07187,2017$

4. Matalatala, M.; Deruyck, M.; Shikhantsov, S.; Tanghe, E.; Plets, D.; Goudos, S.; Psannis, K.E.; Martens, L.; Joseph, W. Multi-Objective Optimization of Massive MIMO 5G Wireless Networks towards Power Consumption, Uplink and Downlink Exposure. Appl. Sci. 2019, 9, 4974

5. Jiayi Zhang, Emil Bjornson,Michail Matthaiou, Derrick Wing Kwan Ng. et. Al., Multiple Antenna Technologies for beyond 5G, arXiV;1910.00092v1[cs.IT],30 Sep 2019

6. Qurrat-Ul-Ain Nadeem, Abla Kammoun, Merouane Debbah,Mohamed Slim Design of 5G Full Dimesion Massive MIMO Systems, Dec. 2016, IEEE Global Communications Conference, Washington DC,USA 
7. Jiarui Wu, Research on Massive MIMO Key Technology in 5G, IOP Conference Series on Materials Science and Engineering 466 (2018)

8. Fuxi Wen, henk Wymeerrsch, Bile Peng, Wee Peng Tay, Hing Cheung So, Diange Yang, A survey on 5G massive MIMO localisation, Digital Signal Processing, 94(2019), 21-28

9. Muhammad Fahad Khan, Kok-Lim Alvin Yau, Rafidah MD.Noor, Survey and taxonomy of clustering, Journal of Network and Computer Applications, 154 (2020)102539

10. Olabode Idown Bismark,Okokpujie Kennedy,Francis Idachaba, Aderemi.A.Atayero, A Primer on MIMO Detection Algorithms for 5G Communication Network, International Journal on Communications Antenna and Propagation, Vol.8,Issue 3,June 2018

11. H. Huang et al., "Deep Learning for Physical-Layer 5G Wireless Techniques: Opportunities, Challenges and Solutions," in IEEE Wireless Communications, vol. 27, no. 1, pp. 214-222, February 2020, doi: 10.1109/MWC.2019.1900027.

12. R A Veer,L C Siddanna Gowd, A Novel Classification Approach for MIMO-OFDM, International Journal of Innovative Technology and Exploring Engineering, Vol.8,Issue 4,Feb. 2019

13. Kamran Zia, Nauman Javed, Muhammad Sial, Sohail Ahmed et.al, A Survey of Coventional and Artificial Intelligence/ Learning based Resource Allocation and Inteference Mitigation Schemes in D2D Enabled Networks, arXiV;1809.08748v1[cs.AI],24 Sept.2018

14. Tareq.M.Shami, David Grace, Alister Burr, John.S.Vardakas, Load Balancing and control with interference mitigation in $5 \mathrm{G}$ heterogeneous networks, EURASIP Journal of Wireless Communications and Networking, $2019(177)$

15. Alexandre Marcastel,E.Veronica Belmega,Panayotis Mertikopoulos, Inbar Fijalkow, Online Interference Mitigation via Learning in Dynamic IoT Environments, IEEE Workshop, Globecom,Dec 2016, Washington DC,USA

16. Ehab Ali, Mahamod Ismail, R.Nordin,G.Abdulah, Beamorming techniques for massive MIMO systems in 5G: overview, classification and trends for future research, Frontiers of Information Technology \& Electronic Engineering, 2017, 18(6), 753-772

17. F. W. Vook, A. Ghosh and T. A. Thomas, "MIMO and beamforming solutions for 5G technology," 2014 IEEE MTT-S International Microwave Symposium (IMS2014), Tampa, FL, 2014, pp. 1-4, doi: 10.1109/MWSYM.2014.6848613.

18. A. A. Kalachikov and N. S. Shelkunov, "Performance Evaluation of Multiuser Beamforming and Nonorthogonal Multiple Access Based on MIMO Channel Measurements," 2019 International Multi-Conference on Engineering, Computer and Information Sciences (SIBIRCON), Novosibirsk, Russia, 2019, pp. 00310034, doi: 10.1109/SIBIRCON48586.2019.8958062.

19. A. M. Elbir and K. V. Mishra, "Deep Learning Design for Joint Antenna Selection and Hybrid Beamforming in Massive MIMO," 2019 IEEE International Symposium on Antennas and Propagation and USNC-URSI Radio Science Meeting, Atlanta, GA, USA, 2019, pp. 1585-1586, doi: 10.1109/APUSNCURSINRSM.2019.8888753.

20. H. P. Tauqir and A. Habib, "Deep Learning Based Beam Allocation in Switched-Beam Multiuser Massive MIMO Systems," 2019 Second International Conference on Latest trends in Electrical Engineering and Computing Technologies (INTELLECT), Karachi, Pakistan, 2019, pp. 1-5, doi: 10.1109/INTELLECT47034.2019.8955466.

21. A. M. Elbir and K. V. Mishra, "Robust Hybrid Beamforming With Quantized Deep Neural Networks," 2019 IEEE 29th International Workshop on Machine Learning for Signal Processing (MLSP), Pittsburgh, PA, USA, 2019, pp. 1-6, doi: 10.1109/MLSP.2019.8918866.

22. K Venugopal, A Alkhateeb, NG Prelcic, RW Heath, Channel estimation for hybrid architecture-based wideband millimeter wave systems, IEEE Journal on Selected Areas in Communications 35 (9), 1996-2009

23. H. Huang, Y. Song, J. Yang, G. Gui and F. Adachi, "Deep-Learning-Based Millimeter-Wave Massive MIMO for Hybrid Precoding," in IEEE Transactions on Vehicular Technology, vol. 68, no. 3, pp. 3027-3032, March 2019, doi: 10.1109/TVT.2019.2893928.

24. T. Maksymyuk, J. Gazda, O. Yaremko and D. Nevinskiy, "Deep Learning Based Massive MIMO Beamforming for 5G Mobile Network," 2018 IEEE 4th International Symposium on Wireless Systems within the International Conferences on Intelligent Data Acquisition and Advanced Computing Systems (IDAACSSWS), Lviv, 2018, pp. 241-244, doi: 10.1109/IDAACS-SWS.2018.8525802.

25. A. Klautau, P. Batista, N. González-Prelcic, Y. Wang and R. W. Heath, "5G MIMO Data for Machine Learning: Application to Beam-Selection Using Deep Learning," 2018 Information Theory and Applications Workshop (ITA), San Diego, CA, 2018, pp. 1-9, doi: 10.1109/ITA.2018.8503086.

26. C. Antón-Haro and X. Mestre, "Learning and Data-Driven Beam Selection for mmWave Communications: An Angle of Arrival-Based Approach," in IEEE Access, vol. 7, pp. 20404-20415, 2019, doi: 10.1109/ACCESS.2019.2895594. 
27. Sneha.V.V, Ismayil Siyad C,S.Tamilselvan, Multilayer Perceptron Scheme for Beamforming and Channel estimation of Massive MIMO, International Journal of Recent Technology and Engineering, Vol.5, Issue 256, July 2019

28. Y. Yang, F. Gao, G. Y. Li and M. Jian, "Deep Learning-Based Downlink Channel Prediction for FDD Massive MIMO System," in IEEE Communications Letters, vol. 23, no. 11, pp. 1994-1998, Nov. 2019, doi: 10.1109/LCOMM.2019.2934851.

29. L. Sanguinetti, A. Zappone and M. Debbah, "Deep Learning Power Allocation in Massive MIMO," 2018 52nd Asilomar Conference on Signals, Systems, and Computers, Pacific Grove, CA, USA, 2018, pp. 12571261, doi: 10.1109/ACSSC.2018.8645343.

30. Motade, S.N.; Kulkarni, A.V. Channel Estimation and Data Detection Using Machine Learning for MIMO 5G Communication Systems in Fading Channel. Technologies 2018, 6, 72.

31. Lin C, Chang Q, Li X. A Deep Learning Approach for MIMO-NOMA Downlink Signal Detection. Sensors. 2019; 19(11):2526.

32. J. Tao, J. Xing, J. Chen, C. Zhang and S. Fu, "Deep Neural Hybrid Beamforming for Multi-User mmWave Massive MIMO System," 2019 IEEE Global Conference on Signal and Information Processing (GlobalSIP), Ottawa, ON, Canada, 2019, pp. 1-5, doi: 10.1109/GlobalSIP45357.2019.8969154.

33. H. P. Tauqir and A. Habib, "Deep Learning Based Beam Allocation in Switched-Beam Multiuser Massive MIMO Systems," 2019 Second International Conference on Latest trends in Electrical Engineering and Computing Technologies (INTELLECT), Karachi, Pakistan, 2019, pp. 1-5, doi: 10.1109/INTELLECT47034.2019.8955466.

34. A. M. Elbir and K. V. Mishra, "Deep Learning Design for Joint Antenna Selection and Hybrid Beamforming in Massive MIMO," 2019 IEEE International Symposium on Antennas and Propagation and USNC-URSI Radio Science Meeting, Atlanta, GA, USA, 2019, pp. 1585-1586, doi: 10.1109/APUSNCURSINRSM.2019.8888753.

35. A. M. Elbir and K. V. Mishra, "Robust Hybrid Beamforming With Quantized Deep Neural Networks," 2019 IEEE 29th International Workshop on Machine Learning for Signal Processing (MLSP), Pittsburgh, PA, USA, 2019, pp. 1-6, doi: 10.1109/MLSP.2019.8918866.

36. Y. Liu, X. Wang, G. Boudreau, A. B. Sediq and H. Abou-zeid, "Deep Learning Based Hotspot Prediction and Beam Management for Adaptive Virtual Small Cell in 5G Networks," in IEEE Transactions on Emerging Topics in Computational Intelligence, vol. 4, no. 1, pp. 83-94, Feb. 2020, doi: 10.1109/TETCI.2019.2926769.

37. A. M. Elbir and K. V. Mishra, "Joint Antenna Selection and Hybrid Beamformer Design Using Unquantized and Quantized Deep Learning Networks," in IEEE Transactions on Wireless Communications, vol. 19, no. 3, pp. 1677-1688, March 2020, doi: 10.1109/TWC.2019.2956146.

38. G. Zhong, K. Zhang, H. Wei, Y. Zheng and J. Dong, "Marginal Deep Architecture: Stacking Feature Learning Modules to Build Deep Learning Models," in IEEE Access, vol. 7, pp. 30220-30233, 2019, doi: 10.1109/ACCESS.2019.2902631.

39. G. Zhong, H. Wei, Y. Zheng and J. Dong, "Marginal Deep Architectures," 2017 4th IAPR Asian Conference on Pattern Recognition (ACPR), Nanjing, 2017, pp. 559-564, doi: 10.1109/ACPR.2017.88.

40. F. C. Ghesu et al., "Marginal Space Deep Learning: Efficient Architecture for Volumetric Image Parsing," in IEEE Transactions on Medical Imaging, vol. 35, no. 5, pp. 1217-1228, May 2016, doi: 10.1109/TMI.2016.2538802.

41. Ahmed Alkhateeb, DeepMIMO: A Generic Deep Learning Dataset for Millimeter Wave and Massive MIMO Applications, arXiv:1902.06435 [cs.IT], Feb. 2019

42. A. Alkhateeb, S. Alex, P. Varkey, Y. Li, Q. Qu and D. Tujkovic, "Deep Learning Coordinated Beamforming for Highly-Mobile Millimeter Wave Systems," in IEEE Access, vol. 6, pp. 37328-37348, 2018, doi: 10.1109/ACCESS.2018.2850226. 